\section{Development of a fast chromotomographic spectrometer}

\author{
Malcolm Gould \\ Stephen Cain \\ Air Force Institute of Technology \\ Department of Electrical Engineering \\ 2950 Hobson Way \\ Building 640 \\ Wright-Patterson AFB \\ Ohio 45433-7765 \\ E-mail: Stephen.Cain@ afit.edu
}

\begin{abstract}
A method for preprocessing spectral information produced by chromotomographic imagers combined with a new postprocessing strategy is proposed. The new technique makes it possible to collect both spectral and limited spatial information from a chromotomographic sensor at kilohertz rates with a conventional charge-coupled device (CCD) array. The proposed technique involves forming a vector projection of each 2-D frame collected by the sensor. The vector projections are postprocessed via a reconstruction algorithm that produces 1-D profiles of the scene and a spectrum for each 1-D pixel. This on-chip preprocessing approach results in a large reduction of the bandwidth through the analog to digital converter, while maintaining the desired temporal resolution of the sensor. High-speed CMOS cameras can obtain comparable speeds, but at significant added cost in hardware and postprocessing time. This makes the proposed sensor attractive for applications where large numbers of sensors are required and the need to obtain spectral information quickly is of paramount importance.
\end{abstract}

[DOI: 10.1117/1.2117007]

Subject terms: hyperspectral; chromotomographic imager.

Paper 050301 LR received Apr. 24, 2005; revised manuscript received Aug. 19, 2005; accepted for publication Sep. 4, 2005; appeared online Sep. 7, 2005; published online Nov. 2, 2005.

\section{Introduction}

The sensor proposed in this paper utilizes a CCD array in conjunction with a direct vision prism in the same way a traditional chromotomographic sensor would, ${ }^{1}$ but unlike a standard chromotomographic imager, it reads out a vector for each frame of data gathered by the sensor instead of the full 2-D image. Vectors are then processed through the 1-D spectral reconstruction algorithm described in this paper to produce the average spectrum of the scene along each row of the CCD array. The projection-based chromotomographic spectrometer has potential application to any remote sensing situation where the target is spatially simple and highly dynamic. The problem of identifying the type of explosive used in an explosive device is an example of a case where the proposed sensor may prove beneficial. The ability to collect spectral information quickly may allow an analysis of the chemical composition of the blast, while reconstruction of one spatial dimension will facilitate the

\footnotetext{
*The opinions and views expressed in this paper are not necessarily those of the Department of Defense or The United States Air Force.

0091-3286/2005/\$22.00
}

estimation of the size of the blast as a function of time. Both of these elements are important for identifying the type and construction of an explosive, ${ }^{2}$ whether it is a standard device or an improvised one. For this reason, the spectra of simulated fireballs will be used to demonstrate the effectiveness of the proposed technique in simulation.

\section{Vector Chromotomographic Modeling and Reconstruction}

A discrete model describing how images are formed by a chromotomographic sensor is shown below. ${ }^{3}$ The actual model for the sensor is understood to be continuous, but in order to facilitate the derivation of a reconstruction algorithm to be used in a digital computer, the discrete approximation to the sensor model will be used:

$i\left(\theta_{k}, x, y\right)=\sum_{m=1}^{M} \sum_{u=1}^{N} \sum_{v=1}^{N} \eta\left(\lambda_{m}\right) o\left(\lambda_{m}, u, v\right) h\left(\lambda_{m}, \theta_{k}, x-u, y-v\right)$,

where the number of pixels on a detector side is $N$ and $\lambda_{m}$ is the wavelength of the light in the center of bin $m$. The variable $i$ describes the average number of photoelectrons collected by a detector located in the array at pixel $(x, y)$ in the CCD array at rotation angle $\theta_{k}$. The true scene $o$ is the average number of photons emanating from the image predicted by geometric optics in the focal plane at pixel coordinates $(u, v)$ integrated over each discrete wavelength bin centered at $\lambda_{m}$. The values of $\theta_{k}$ are specific angles the direct vision prism rotates to at $K$ different frame acquisition times. $h$ is the point spread function of the optical system as a function of wavelength index $\lambda_{m}$, rotation angle $\theta_{k}$, and position in the CCD array. The normalized point spread function $h$ is precomputed from knowledge of the aberrations in the optical system and dispersion through the direct vision prism via a Rayleigh-Sommerfeld diffraction calculation at each wavelength. ${ }^{4} \eta$ is the average quantum efficiency of each detector in the array as a function of wavelength times the atmospheric transmission and optics transmission.

The signals measured by the CCD at each sample value in units of electrons are assumed to contain the average signal collected by the array, $i$ plus noise. The projectionbased chromotomographic sensor will serve to integrate the charge along each row of the array to produce a vector of sampled outputs at each rotation angle associated with a readout time of the sensor. Assuming that the point spread function is shift-invariant and setting $o_{y}\left(v, \lambda_{m}\right)$ $=\sum_{u=1}^{N} o\left(u, v, \lambda_{m}\right)$ and $h_{y}\left(\lambda_{m}, \theta_{k}, y\right)=\sum_{x=1}^{N} h\left(\lambda_{m}, \theta_{k}, x, y\right)$, the following convolution operation between the row summed hyperspectral cube $o_{y}$ and the row summed point spread function $h_{y}$ is related to the summation $i_{y}\left(\theta_{k}, y\right)$ $=\sum_{x=1}^{N} i\left(\theta_{k}, x, y\right)$ via

$i_{y}\left(\theta_{k}, y\right)=\sum_{m=1}^{M} \eta\left(\lambda_{m}\right) \sum_{v=1}^{N} o_{y}\left(v, \lambda_{m}\right) h_{y}\left(\lambda_{m}, \theta_{k}, y-v\right)$.

The projection operation on the CCD accounts for the dramatic speed increase possible with projection-based technique. If for example a $250-\times 250$-pixel CCD array is used in conjunction with an analog-to-digital (A/D) converter capable of producing 3 million samples per second, 


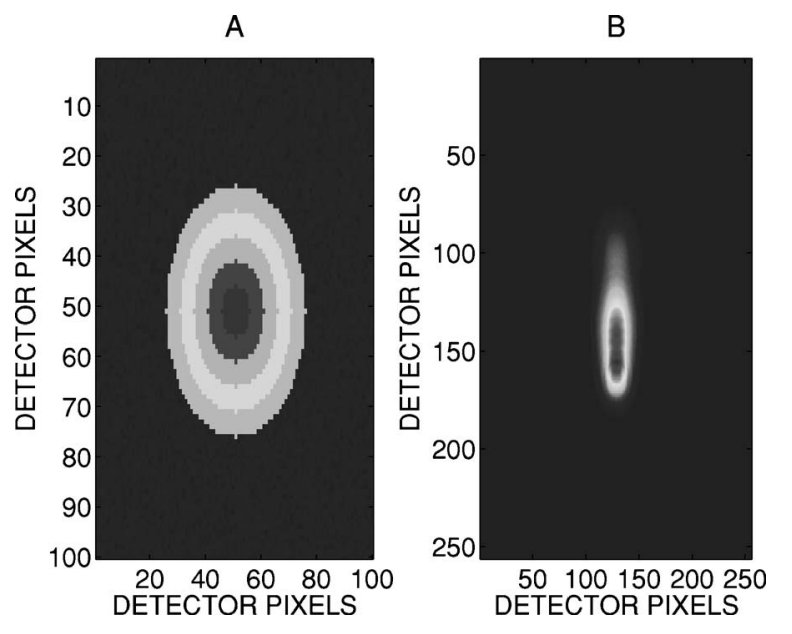

Fig. 1 (A) shows the temperature distribution of the fireball mapped onto detector pixels projected out at the source plane. (B) shows the dispersed image formed on the surface of the CCD array at a DVP rotation angle of 0 deg.

then the camera could read out approximately 48 full frame images per second. When the data are compressed on the $\mathrm{CCD}$ into vectors, then the vector readout rate would be $12 \mathrm{kHz}$. The compression of the image into vectors is accomplished by accomplishing $N$ parallel shifts prior to execution of the serial shifts that move the charge to the readout amplifier to be digitized.

It is assumed that the observations at each detector on the $\mathrm{CCD}$ array are independent Poisson random variables whose mean is the average number of photoelectrons expected at that pixel $i .^{5}$ The summation will in turn produce Poisson random variables at the output of the system whose mean is $i_{y}$. Using this statistical model, the probability mass function (PMF) for a set of $K$ vectors will be:

$$
P\left[d=D \forall y, \theta_{k}\right]=\prod_{y=1}^{N} \prod_{k=1}^{K} \frac{i_{y}\left(\theta_{k}, y\right)^{d_{y}\left(\theta_{k}, y\right)} e^{-i_{y}\left(\theta_{k}, y\right)}}{d_{y}\left(\theta_{k}, y\right) !},
$$

where $d$ is a particular realization of the random variable $D$. Using this PMF, a maximum-likelihood estimator for $o_{y}$ can be formed by maximizing this PMF with respect to $o_{y}$. This can be accomplished by maximizing the natural logarithm of the PMF. Using a procedure similar to the one developed by Richardson, ${ }^{7}$ an iterative algorithm of the following form is devised:

$o_{y}^{\text {new }}\left(\lambda_{o}, v_{o}\right)=o_{y}^{o l d}\left(\lambda_{o}, v_{o}\right) \sum_{y=1}^{N} \sum_{k=1}^{K} \frac{d\left(\theta_{k}, y\right)}{i_{y}\left(\theta_{k}, y\right)} h_{y}\left(\lambda_{o}, \theta_{k}, y-v_{o}\right)$.

The iterations begin by setting $o_{y}\left(\lambda_{m}, v\right)=1$ and continue until $\quad\left\{\sum_{y=1}^{N} \Sigma_{k=1}^{K}\left[d\left(\theta_{k}, y\right)-i\left(\theta_{k}, y\right)\right]^{2}\right\}<\left[\Sigma_{y=1}^{N} \Sigma_{k=1}^{K} d\left(\theta_{k}, y\right)\right]$. This criterion forces the estimated variance to be on the order of the mean.

\section{System Performance}

The simulated chromotomographic system possesses a direct vision prism (DVP) made from $\mathrm{LiF}$ and $\mathrm{BaF}_{2}$ imaging onto a $256-\times 256$-pixel InSb detector array through optics with an $f$ number of 10 . This $f$ number is consistent with an
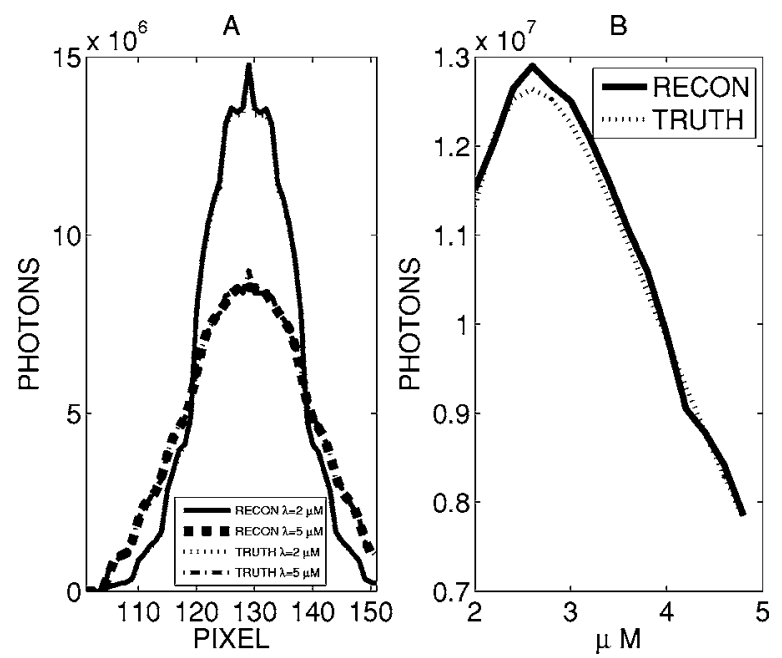

Fig. 2 (A) shows reconstructed profiles of the fireball at wavelengths of $2 \mu \mathrm{m}$ and $5 \mu \mathrm{m}$, respectively, versus the true profile at those wavelengths. (B) shows the reconstructed spectrum for a vector sample at the center of the fireball versus the true spectrum.

optical system that might be carried by a tactical aircraft or unmanned aerial vehicle having a 4-in. entrance aperture and an effective focal length of $1 \mathrm{~m}$. An atmospheric transmission profile is used that contains extinction bands at 2.6 and $4.4 \mu \mathrm{m}^{8}$ and a transmission filter window is used in the system to restrict the incoming radiation to be between 2 and $5 \mu \mathrm{m}$. A midwave infrared sensor is chosen for this simulation because it would be sensitive to thermal events whose high temperature would produce significant radiation and good contrast against background radiation and solar reflection in the 2- to $5-\mu \mathrm{m}$ band. The target is a fireball at a range of $3 \mathrm{~km}$ with concentric rings of varying temperature ranging from $1600 \mathrm{~K}$ to $800 \mathrm{~K}$ with a random background around the fireball at a $300 \mathrm{~K}$ mean and a 10deg standard deviation shown in Fig. 1.

The target is both thermally and structurally similar to that of a bomb detonation fireball, while the background temperature and distribution is meant to approximate a room temperature thermal background with clutter. The speed of the detonation would require a sensor that can rapidly acquire the light emitted from the explosion. If the sensor collects the data too slowly, an accurate spectral signature would be difficult to obtain. In this application the projection-based chromotomographic sensor would be used to measure the spectrum of the fireball so that it could be analyzed to determine the type of ordinance used in the explosion in the event of an attack or if any secondary explosions occurred in a bomb damage assessment application. An observation distance of $3 \mathrm{~km}$ is chosen to approximate the range at which a sensor on an unmanned aerial vehicle or tactical aircraft might view the explosion. The projection-based spectral reconstruction algorithm was used to process the simulated data that contained the dispersion of the instrument and Poisson noise. Figure 2 shows recovered profiles of the fireball at wavelengths of $2 \mu \mathrm{m}$ and $5 \mu \mathrm{m}$, respectively, demonstrating the quality of the reconstruction at opposite ends of the spectrum. 


\section{OE LETTERS}

\section{Conclusions}

The simulated data and results show the ability of the projection-based chromotomographic sensor and reconstruction algorithm to measure the spectrum of a geometrically simple target. The potential of the proposed sensor to gather spectral data quickly in cases where spatial information is of secondary importance yields an attractive alternative to less photon efficient spectral separation methods such as grating spectrometers. Another advantage of the projection-based chromotomographic sensor is it differs from a traditional 2-D chromotomographic imager only in software. This would allow the possibility of switching between 1-D high-speed spectroscopy and 2-D chromotomographic imaging with the same sensor payload. Future research will attempt to characterize the performance of the algorithm on a temporally dynamic target, quantify the spectral resolution of the proposed sensor, and apply the technique to real data.

\section{References}

1. J. M. Mooney, "Angularly multiplexed spectral imager," Proc. SPIE 2480, 65-77 (1995).

2. J. A. Orson, W. F. Bagby, and G. P. Perram, "Infrared signatures from bomb detonations," Infrared Phys. Technol. 44, 101-107 (2003).

3. A. K. Brodzik and J. M. Mooney, "Convex projections algorithm for restoration of limited-angle chromotomographic images," J. Opt. Soc. Am. A 16, 246-257 (1999).

4. J. W. Goodman, Introduction to Fourier Optics, McGraw-Hill, New York (1968).

5. J. W. Goodman, Statistical Optics, John Wiley and Sons, New York (1985).

6. H. L. Van Trees, Detection, Estimation and Modulation Theory, John Wiley and Sons, New York (1968).

7. B. H. Richardson, "Bayesian-based iterative method of image restoration," J. Opt. Soc. Am. 62, 55-59 (1972).

8. S. D. Lord, "A new software tool for computing Earth's atmospheric transmission in the near and far infrared radiation," Technical Report, NASA Tech. Memo. 103957, Gemini Observatory, Mauna Kea, Hawaii (1992). 